\title{
Survivin gene expression increases gastric cancer cell lymphatic metastasis by upregulating vascular endothelial growth factor-C expression levels
}

\author{
JUNYAN ZHANG $^{1}, \mathrm{ZHI} \mathrm{ZHU}^{1}, \mathrm{ZHE} \mathrm{SUN}^{1}, \mathrm{XUREN} \mathrm{SUN}^{2}, \mathrm{ZHENNING} \mathrm{WANG}^{1}$ and HUIMIAN XU ${ }^{1}$ \\ Departments of ${ }^{1}$ Surgical Oncology and ${ }^{2}$ Gastroenterology, First Affiliated Hospital, China Medical University, \\ Shenyang, Liaoning 110001, P.R. China
}

Received May 27, 2013; Accepted November 14, 2013

DOI: $10.3892 / \mathrm{mmr} .2013 .1858$

\begin{abstract}
The aim of this study was to investigate the correlation between the expression of survivin and vascular endothelial growth factor-C (VEGF-C) in gastric cancer and the pathway by which survivin may affect gastric cancer lymphatic metastasis. The study may provide novel targets for treating gastric cancer lymphatic metastasis and distal dissemination. Survivin and VEGF-C expression in gastric carcinoma and peri-carcinoma ( $2 \mathrm{~cm}$ away from the carcinoma) tissues, obtained from 195 patients who underwent curative gastrectomy surgery (130 cases presented with lymph node metastasis and 65 cases presented without metastasis), was examined immunohistochemically using a tissue microarray. Plasmids containing survivin and VEGF-C shRNA were constructed and transfected into SGC-7901 gastric cancer cells. The expression levels of the two genes were examined using western blot analysis and $\mathrm{qPCR}$, and the results were statistically analyzed. The expression levels of survivin and VEGF-C were 51.3 and $55.4 \%$, respectively, in gastric carcinoma. Survivin and VEGF-C were located mainly in the cytoplasm of the tumor cells. The expression levels of survivin and VEGF-C were significantly higher in patients with lymph node metastasis than in those without metastasis ( $\mathrm{P}=0.008$ and 0.001 , respectively). Patients with high expression levels of survivin and VEGF-C showed significantly less favorable survival rates compared with patients with low expression levels of those two genes $(\mathrm{P}=0.003$ and 0.039 , respectively). Moreover, patients with co-expression of the two genes usually had a poorer prognosis $(\mathrm{P}=0.003)$. However, multivariate analysis demonstrated that neither of the two genes were an independent prognostic determinant. The levels of VEGF-C expression may be regulated
\end{abstract}

Correspondence to: Dr Huimian Xu, Department of Surgical Oncology, First Affiliated Hospital, China Medical University, 155 North Nanjing Street, Shenyang, Liaoning 110001, P.R. China

E-mail: xuhuimian@126.com

Key words: survivin, VEGF-C, gastric cancer, lymphatic metastasis by survivin expression. Conversely, inhibiting VEGF-C gene expression by shRNA did not reduce survivin expression at the mRNA or protein levels. Survivin and VEGF-C were expressed in gastric cancer cells and were significantly associated with lymphatic metastasis. Survivin may be a regulator of VEGF-C expression in gastric cancer cells, and is essential in invasion and lymphatic metastasis. Moreover, survivin may be able to serve as a chemotherapy target for gastric cancer.

\section{Introduction}

Despite the overall decreasing incidence and mortality rates observed over the last 50 years, gastric cancer ranks as the fourth most common malignancy and the second leading cause of cancer-related mortality worldwide (1). Approximately one million novel cases of gastric cancer and $>700,000$ deaths resulting from it were reported in 2008, with an overall five-year survival rate of $<20 \%$ (1). Lymphatic metastasis and distal dissemination are the main causes of mortality. Metastasis and recurrence following surgery are the main factors affecting the therapeutic efficacy of gastric cancer. Regional lymph nodes are the most common site of metastasis and lymph node metastasis is a major prognostic factor in gastric cancer. Understanding the mechanisms of lymphatic metastasis represents a crucial step and may result in a novel therapeutic target in the treatment of human gastric cancer.

Numerous pathways have been proposed to be involved in the process of gastric cancer lymphatic metastasis. A number of markers, such as epidermal growth factor receptor (EGFR) (2) and cyclooxygenase 2 (COX-2) (3), have been detected as predicted targets of gastric cancer lymphatic metastasis. Certain studies have indicated that survivin and vascular endothelial growth factor-C (VEGF-C) may be involved in lymphatic metastasis of gastric cancer $(4,5)$.

Survivin is a protein of 142 amino acids and is the smallest mammalian member of the inhibitor of apoptosis protein family (6). It regulates two essential cellular processes by inhibiting apoptosis and promoting cell proliferation. Survivin is expressed at high levels during fetal development, while it is rarely expressed in normal healthy adult tissues. Overexpression of survivin has been observed in a number of malignant tumors, including carcinomas of the lung, breast, stomach, colon and 
ovary, as well as melanomas and lymphomas (7). Expression of survivin is also associated with worse therapy results, higher risk of recurrences and resistance to chemotherapy $(8,9)$. Several studies have indicated that the expression of survivin is significantly associated with the occurrence of lymphatic metastasis, but the mechanism by which survivin controls lymphatic metastasis remains unknown $(10,11)$.

VEGF-C is a member of the VEGF family. Studies have demonstrated that VEGF-C is critical in facilitating tumor metastasis $(12,13)$. VEGF-C induces lymphangiogenesis and lymph node metastasis of tumors by activating VEGFR-3 in lymphatic endothelial cells. It has been demonstrated that VEGF-C is strongly expressed and is an important predictor of lymphangiogenesis and prognosis in numerous types of cancer, including gastric carcinoma $(14,15)$.

There are few studies concerned with the functions of survivin and VEGF-C and their clinical characteristics in gastric cancer. In the present study, the correlation between the expression of survivin and VEGF-C, and its association with lymphangiogenesis in gastric cancer tissues was investigated. The pathway by which survivin may affect gastric cancer lymphatic metastasis was also predicted.

\section{Material and methods}

Patients and specimens. The study enrolled 195 patients (139 males and 56 females; age range, 30-81 years; average age, 58 years) who underwent surgery (total or partial gastrectomy) for histologically proven gastric carcinoma between 2006 and 2008 at the Department of Surgical Oncology, First Hospital of China Medical University (Shenyang, China). Tumor-node-metastasis staging was conducted according to the American Joint Committee on Cancer classification, and histological grading was performed according to World Health Organisation criteria (16). The clinical information obtained from the records and the histopathology reports included patient age, first diagnosis, tumor size and grade, and lymph node involvement. Follow-up data were available from all patients, whom were assessed every six months for five years or until they succumbed to the disease during those five years. The study was approved by the Ethics Committee of China Medical University (Shenyang, China) and written informed consent was obtained from the patients family.

Tissue microarray construction. The paraffin tissue microarray (TMA; Outdo Biotech, Co., Ltd., Shanghai, China) was constructed according to the guidelines previously used by the National Cancer Institute's Tissue Array Research Programme. Each individual case was represented by one tumor core $(1 \mathrm{~mm})$ and one peri-carcinoma core $(1 \mathrm{~mm})$ that was obtained from the original paraffin block (from the archive of the Institute of Pathology, First Hospital of China Medical University) according to its hematoxylin and eosin (H\&E) slides. These core needle biopsies were placed in recipient paraffin array blocks at defined coordinates. The cores were incubated in the paraffin block for $30 \mathrm{~min}$ at $37^{\circ} \mathrm{C}$ to improve adhesion between the cores and the paraffin of the recipient block. The paraffin tissue microarray blocks were then cut into 5- $\mu \mathrm{m}$ sections by an ultra-thin semi-automatic microtome (RM2235; Leica Biosystems, Wetzlar, Germany).
Immunohistochemical staining. Specimens were immunostained with the standard labeled streptavidin-biotin (LsAB) protocol. The $5-\mu \mathrm{m}$ sections from each paraffin block were deparaffinized and rehydrated. TMA slides were heated in sodium citrate buffer (0.01 mol/l, $\mathrm{pH}$ 6.0) for $15 \mathrm{~min}$ in a microwave oven. Following cooling for $20 \mathrm{~min}$ and washing in phosphate-buffered saline (PBS), endogenous peroxidase was detected by incubating samples with PBS containing $10 \%$ normal goat serum (Envision ${ }^{\mathrm{TM}}+$ kit; Dako , Glostrup, Denmark) for $30 \mathrm{~min}$. The sections were then incubated with each primary antibody overnight at $4^{\circ} \mathrm{C}$. The primary antibodies were anti-survivin (mouse monoclonal; 1:10; Santa Cruz Biotechnology, Inc., Santa Cruz, CA, USA) and anti-VEGF-C (rabbit polyclonal; 1:200; Abcam, Cambridge, MA, USA). A further wash in PBS was followed by treatment with a peroxidase-labeled polymer conjugated to goat anti-mouse or anti-rabbit immunoglobulins (Envison ${ }^{\mathrm{TM}}+$ kit; Dako) as the secondary antibody for $10 \mathrm{~min}$ at room temperature. The staining was visualized with diaminobenzidine, followed by counterstaining with hematoxylin. As a negative control, PBS was substituted for the primary antibody.

Evaluation of immunohistochemical staining. The immunohistochemical score, based on the German immunoreactive score, was used for survivin and VEGF-C immunohistochemical evaluation. Two pathologists, who were blinded to the outcome data, independently evaluated the sections three times. The staining of survivin and VEGF-C was then scored from 0 to 3, considering only the cytoplasmic reaction: A score of 0 required $<10 \%$ of the cells to be stained, 1 required $11-20 \%$ of cells to be stained, 2 required $21-50 \%$ of cells to have been stained and 3 required $51-100 \%$ of cells to be stained.

Cell culture. The gastric cancer cell line, SGC-7901, was purchased from the Cell Bank of Type Culture Collection of the Chinese Academy of Sciences (Shanghai, China) and cultured in RPMI-1640 medium (Hyclone, Logan, UT, USA) supplemented with $10 \%$ fetal bovine serum (FBS) in a $5 \% \mathrm{CO}_{2}$ humidified atmosphere at $37^{\circ} \mathrm{C}$. Log phase cells were collected following trypsin digestion by centrifugation for $5 \mathrm{~min}$ at $33.3 \mathrm{x}$ g, re-suspended in PBS and counted using a hemocytometer (Hausser Scientific, Horsham, PA, USA).

Construction and transfection of plasmid-shRNA. Plasmids containing survivin-shRNA and VEGF-C-shRNA were constructed by Shanghai GenePharma Co., Ltd. (Shanghai, China).First, $10 \mu$ g plasmid-survivin-shRNA and $10 \mu$ g plasmidVEGF-C-shRNA, respectively and $25 \mu$ l Lipofectamine 2000 (Invitrogen Life Technologies, Carlsbad, CA, USA) were mixed together with $1350 \mu 1$ RPMI-1640 medium (without FBS), and then the mixture was transfected into SGC-7901 gastric cancer cells. The mixture was added into a $25-\mathrm{cm}^{2}$ culture flask that was previously plated with $1 \times 10^{6}$ SGC-7901 gastric cancer cells. The culture medium was replaced with complete RPMI-1640 medium $6 \mathrm{~h}$ following inoculation and the cells were collected after a further $28 \mathrm{~h}$. Protein and RNA were extracted for western blot and qPCR analyses, respectively.

Western blot analysis. Proteins were separated by SDS-PAGE, transferred onto nitrocellulose membranes and detected using 
the relevant primary and secondary antibodies. Primary antibodies included: Anti-survivin (mouse monoclonal, Santa Cruz Biotechnology, Inc.), anti-VEGF-C (rabbit polyclonal, Abgent, San Diego, CA, USA) and anti- $\beta$-actin (mouse monoclonal, Santa Cruz Biotechnology, Inc.).

RNA isolation and $q P C R$. Total RNA was extracted using the guanidinium thiocyanate-phenol-chloroform method (17). RNA yield and purity were determined photometrically (BioPhotometer, Eppendorf, Hamburg, Germany), and reverse transcription was performed. Survivin and VEGF-C were amplified using qPCR. Primer subsequences were as follows: Forward: 5'-ACAGTCCATGCCATCACTGCC-3' and reverse: 5'- GCCTGCTTCACCACCTTCTTG-3' for $\beta$-actin; forward: 5'-TCATAGAGCTGCAGGGTGGATTGT-3' and reverse: 5'-AGTAGGGTCCACAGCAGTGTTTGA-3' for survivin; and forward: 5'-AACCTCCATGTGTGTCCGTC-3' and reverse: 5'-TGGCAAAACTGATTGTTACTGG-3' for VEGF-C. A total of $10 \mathrm{ng}$ of reverse-transcribed total RNA was used as the template, and the PCR reaction contained $20 \mathrm{pmol} / \mathrm{ml}$ of each forward and reverse primer and SYBR Premix Ex Taq II (Takara Bio, Inc., Dalian, China) in a final volume of $20 \mu \mathrm{l}$. An ABI PRISM 7700 Sequence Detection system (Applied Biosystems, Inc., Foster City, CA, USA) was used for the amplification. Cycling conditions consisted of an initial denaturation step at $95^{\circ} \mathrm{C}$ for $10 \mathrm{~min}$ as a 'hot start', followed by 40 cycles of $95^{\circ} \mathrm{C}$ for $15 \mathrm{sec}$, annealing temperature $\left(72^{\circ} \mathrm{C}\right)$ for $30 \mathrm{sec}$, and a final extension at $72^{\circ} \mathrm{C}$ for $10 \mathrm{~min}$. GAPDH was used in each experiment as an endogenous control. The relative quantification for a gene was expressed as fold changes over the control group. Fold changes were calculated using the $2^{-\Delta \Delta C t}$ method.

Statistical analysis. Kaplan-Meier analysis was applied to estimate cancer-specific survival. Different groups were compared using the log-rank test. Univariate analysis used the $\chi^{2}$ test or a two-tailed t-test for statistical comparisons. Multivariate analysis was conducted with the Cox regression model. Statistical procedures were analyzed with SPSS software, version 16.0 (SPSS, Inc., Chicago, IL, USA). P $<0.05$ was considered to indicate a statistically significant difference.

\section{Results}

Expression of survivin and VEGF-C proteins. Cancer tissue exhibited positive cytoplasmic staining for survivin (100 cells, 51.3\%) and VEGF-C (108 cells, 55.4\%). Gastric carcinoma and peri-carcinoma tissues positively expressed the two proteins. The proteins showed as a yellow or brown-yellow stain in the cytoplasm of the carcinoma cells (Fig. 1). Survivin and VEGF-C were expressed at significantly higher levels in patients with lymphatic metastasis $(\mathrm{P}=0.008$ and 0.001 , respectively). Survivin and VEGF-C were expressed at significantly higher levels in patients with lymphatic metastasis $(\mathrm{P}=0.008$ and 0.001 , respectively). The survivin expression levels were also significantly different in tumors of different histological types $(\mathrm{P}=0.013)$, and the VEGF-C expression levels were significantly different in patients with different ages. However, there were no significant differences in the expression levels of the two genes in Lauren's classifications, tumor locations and sizes (Table I).

\section{Survival analysis}

Univariate prognostic analyses. According to the follow-up data, 78 of the 195 assessable cases did not survive. The overall survival (OS) rate for patients was $60.0 \%$. Analysis of the impact of survivin status is shown in Fig. 2A. Among the patients with low survivin expression levels, 27 cases had died and the OS rate was $71.6 \%$, whereas 51 cases had died in the group of patients with high expression levels of survivin and the OS rate was $49.0 \%$. Patients with high survivin expression levels tended to have a poorer prognosis than the patients with low survivin expression levels $(\mathrm{P}=0.003, \log$-rank test). The OS rates of patients with low and high VEGF-C expression levels were 66.7 and $54.6 \%$, respectively. Kaplan-Meier curves of overall survival stratified by VEGF-C status are shown in Fig. 2B. Patients with high VEGF-C expression tended to have a poorer prognosis than patients with low VEGF-C expression ( $\mathrm{P}=0.039$, log-rank test).

In the patients with co-expression of survivin and VEGF-C, the OS rate was lower than that in the patients with single or no expression of survivin and VEGF-C $(\mathrm{P}=0.003)$ (Fig. 2C). This result indicated that patients with co-expression of survivin and VEGF-C tended to have the poorest prognosis compared with patients with single or no expression.

Multivariate analysis and Cox proportional hazard regression model. In Cox regression for OS rate, including patient age, lymph node metastasis, histological differentiation, $\mathrm{T}$ stage, and survivin and VEGF-C expression, only lymph node metastasis $(\mathrm{P}<0.001$; hazard ratio, $1.425 ; 95 \%$ confidence interval, 1.328-1.529) and $\mathrm{T}$ stage $(\mathrm{P}<0.001$; hazard ratio, 1.340; 95\% confidence interval, 1.181-1.522) remained as independent prognostic factors. Neither of the two genes was an independent prognostic factor (survivin: $\mathrm{P}=0.684$; hazard ratio, 1.181; confidence interval, 0.894-1.076; VEGF-C: $\mathrm{P}=0.116$; hazard ratio, 1.215; confidence interval, 0.935-1.650).

Expression levels of survivin and VEGF-C. According to the immunohistochemical staining results, there was a significant difference between the levels of survivin and VEGF-C expression $(\mathrm{P}<0.05)($ Table II). When the levels of survivin expression increased from degree zero to three, the levels of VEGF-C expression increased correspondingly. The Pearson coefficient of contingency was $\mathrm{C}=0.514$, which implies there is a true correlation between survivin and VEGF-C expression.

In the SGC-7901 gastric cancer cells, when survivin was downregulated, VEGF-C was downregulated, as shown by the western blot analysis, and when survivin was upregulated, VEGF-C was upregulated correspondingly. qPCR demonstrated that the levels of VEGF-C mRNA positively correlated with the levels of survivin mRNA. However, the results did not reveal whether the levels of survivin expression may be regulated by VEGF-C expression using western blot and qPCR analysis (Fig. 3).

\section{Discussion}

Survivin is expressed in nearly all human malignancies as well as embryonic and fetal tissues, but is almost undetectable in normal adult tissues (17). Overexpression of survivin in cancer invariably provides a survival advantage in tumor cells. As a result, survivin has been considered as a potential 
Table I. Correlation between VEGF-C and survivin expression and clinicopathological factors of gastric cancer.

\begin{tabular}{|c|c|c|c|c|c|c|}
\hline \multirow[b]{2}{*}{ Variables } & \multicolumn{2}{|c|}{ VEGF-C protein expression (n) } & \multirow[b]{2}{*}{$\mathrm{P}$-value } & \multicolumn{2}{|c|}{ Survivin protein expression (n) } & \multirow[b]{2}{*}{ P-value } \\
\hline & Negative & Positive & & Negative & Positive & \\
\hline Patients (n) & 87 & 108 & & 95 & 100 & \\
\hline \multicolumn{7}{|l|}{ Gender } \\
\hline Male & 59 & 80 & \multirow[t]{2}{*}{0.337} & 70 & 69 & \multirow[t]{2}{*}{0.470} \\
\hline Female & 28 & 28 & & 25 & 31 & \\
\hline \multicolumn{7}{|l|}{ Age (years) } \\
\hline$\leq 60$ & 59 & 57 & \multirow[t]{2}{*}{0.033} & 62 & 54 & \multirow[t]{2}{*}{0.109} \\
\hline$>60$ & 28 & 51 & & 33 & 46 & \\
\hline \multicolumn{7}{|l|}{ Tumor location } \\
\hline Cardia & 12 & 12 & \multirow[t]{3}{*}{0.492} & 9 & 15 & \multirow[t]{3}{*}{0.443} \\
\hline Body & 9 & 7 & & 9 & 7 & \\
\hline Antrum & 66 & 89 & & 77 & 78 & \\
\hline \multicolumn{7}{|l|}{ Tumor size (cm) } \\
\hline$\leq 4$ & 46 & 50 & \multirow[t]{2}{*}{0.361} & 52 & 44 & \multirow[t]{2}{*}{0.134} \\
\hline$>4$ & 41 & 58 & & 43 & 56 & \\
\hline \multicolumn{7}{|l|}{ Lauren's classification } \\
\hline Intestinal type & 38 & 53 & \multirow[t]{2}{*}{0.453} & 49 & 42 & \multirow[t]{2}{*}{0.180} \\
\hline Diffuse type & 49 & 55 & & 46 & 58 & \\
\hline \multicolumn{7}{|l|}{ Histological type } \\
\hline Well-differentiated & 38 & 47 & \multirow[t]{2}{*}{0.982} & 50 & 35 & \multirow[t]{2}{*}{0.013} \\
\hline Poorly differentiated & 49 & 61 & & 45 & 65 & \\
\hline \multicolumn{7}{|c|}{ Lymphovascular invasion } \\
\hline Yes & 20 & 29 & \multirow[t]{2}{*}{0.536} & 24 & 25 & \multirow[t]{2}{*}{0.966} \\
\hline No & 67 & 79 & & 71 & 75 & \\
\hline \multicolumn{7}{|l|}{ T stage } \\
\hline $\mathrm{T} 1$ & 8 & 10 & \multirow[t]{4}{*}{0.653} & 10 & 8 & 0.050 \\
\hline $\mathrm{T} 2$ & 18 & 15 & & 22 & 11 & \\
\hline $\mathrm{T} 3$ & 57 & 78 & & 61 & 74 & \\
\hline $\mathrm{T} 4$ & 4 & 5 & & 2 & 7 & \\
\hline $\mathrm{N}$ stage & & & & & & \\
\hline No & 39 & 26 & 0.001 & 42 & 23 & 0.008 \\
\hline N1 & 20 & 18 & & 19 & 19 & \\
\hline $\mathrm{N} 2$ & 12 & 19 & & 12 & 19 & \\
\hline N3 & 16 & 45 & & 22 & 39 & \\
\hline
\end{tabular}

Table II. Correlation between survivin and VEGF-C expression in study patients $(n=95)$.

\begin{tabular}{lccrc}
\hline & VEGF-C (0) (n) & VEGF-C (1+) (n) & VEGF-C (2+) (n) & VEGF-C (3+) (n) \\
\hline Survivin (0) & 29 & 14 & 21 & 17 \\
Survivin (1+) & 16 & 17 & 9 & 3 \\
Survivin (2+) & 13 & 12 & 16 & 9 \\
Survivin (3+) & 3 & 2 & 8 & 6 \\
\hline
\end{tabular}

$0,<10 \%$ of cells; $1,11-20 \%$ of cells stained: $2,21-50 \%$ of cells stained; 3 , required $51-100 \%$ of cells stained. VEGF-C, vascular endothelial growth factor-C.

tumor marker and an important therapeutic target (18). Studies on different types of tumors have indicated that the expression of survivin correlates with lymphatic metastasis (19-21). However, few studies have focused on the mechanisms by which survivin protein expression is regulated in gastric cancer. The results of the present study suggest that survivin may influence gastric cancer lymphatic metastasis and distal invasion through VEGF-C.

VEGF-C, a key factor for angiogenesis, interacts with the VEGF-3 receptor to promote hyperplasia of lymphatic vessels. A number of studies have indicated that the upregulation of VEGF-C promotes tumor lymphatic vessel formation 


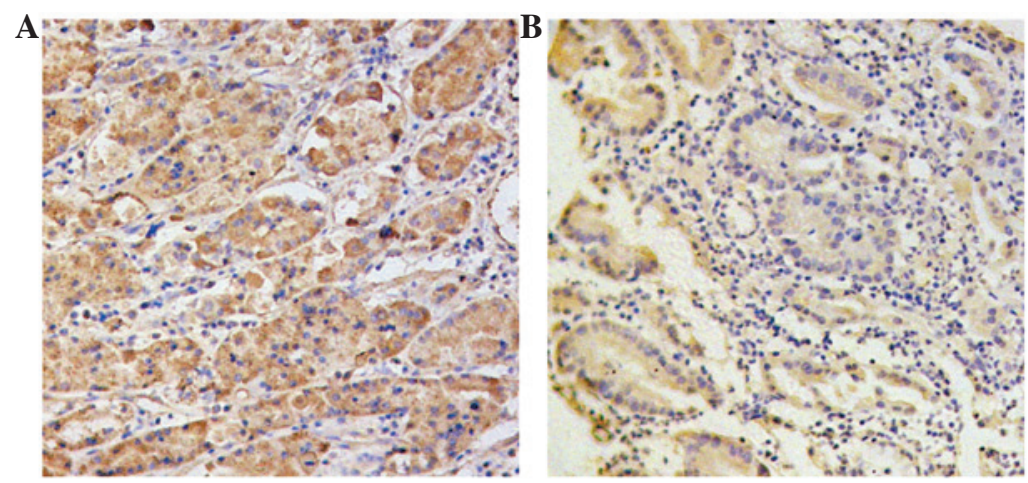

Figure 1. Immunohistochemical staining of survivin and VEGF-C in gastric carcinoma. (A) The positive expression of (A) survivin protein and (B) VEGF-C stained as yellow or brown-yellow in the cytoplasm of the carcinoma cells. (LsAB; magnification, x200). VEGF-C, vascular endothelial growth factor-C; LsAB, labeled streptavidin-biotin.
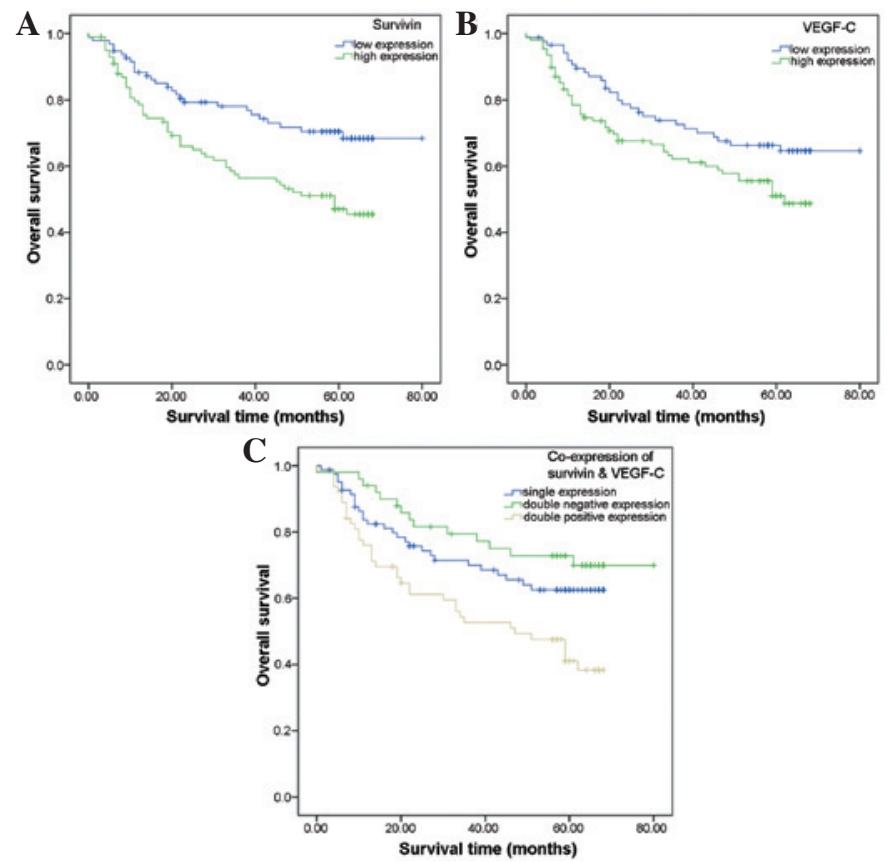

Figure 2. Kaplan-Meier OS curves for 195 patients with gastric carcinoma. (A) Patients with positive survivin expression had a significantly worse OS rate compared with those who were survivin-negative ( $\mathrm{P}=0.003$, log-rank test). (B) Patients with positive VEGF-C expression had a significantly worse OS rate compared with those who were VEGF-C-negative ( $\mathrm{P}=0.039$, log-rank test). (C) Patients with co-expression of survivin and VEGF-C had a significantly worse OS rate compared with those with single or no expression of survivin and VEGF-C ( $\mathrm{P}=0.003, \log -$ rank test). OS, overall survival; VEGF-C, vascular endothelial growth factor-C.

A

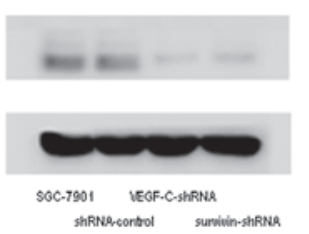

C

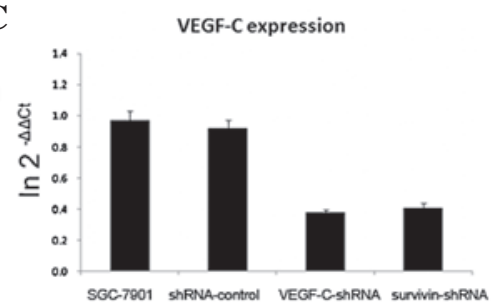

VEGF-C

$\beta$-actin
B
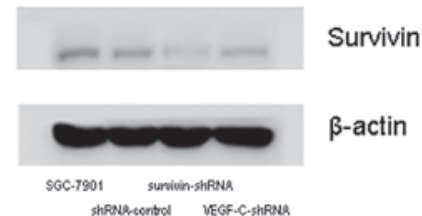

D

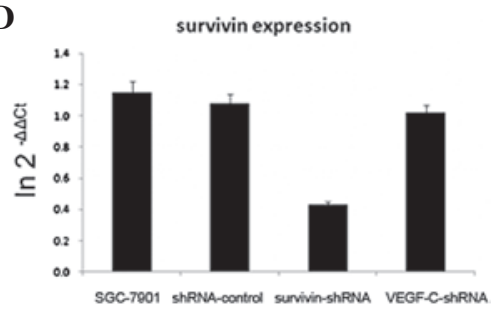

Figure 3. Expression of survivin and VEGF-C in differently processed cells at the protein and mRNA level. (A and B) Western blot analysis for protein expression. (C and D) qPCR for mRNA expression. VEGF-C, vascular endothelial growth factor-C. 
and increases lymph node metastasis. VEGF-C is vital for the lymphangiogenic process, supported by evidence from transgenic and gene deletion animal models through the VEGF-C/VEGFR-3 axis (23,24). 'VEGF-C has been demonstrated to be highly expressed in gastric carcinoma, and has a negative influence on prognosis and a positive correlation with lymph node metastasis $(15,24)$. The results of the present study demonstrated that primary gastric carcinoma tissue exhibited elevated expression levels of VEGF-C, and there was a significant association between VEGF-C expression and lymphatic metastasis.

In the present study, survivin and VEGF-C were expressed at higher levels in patients with lymphatic metastasis. The survivin expression levels were also statistically different in tumors of differing histological type and T stage. Patients with high survivin or VEGF-C expression levels tended to have a poorer prognosis. Moreover, in comparison with patients that had low expression levels of survivin and VEGF-C (S-C-), the patients with high expression levels of survivin and VEGF-C $(\mathrm{S}+\mathrm{C}+)$ had a poorer $\mathrm{OS}$ rate. These results suggest that gastric cancer co-expressing survivin and VEGF-C has higher levels of angiogenesis and lymphangiogenesis, and higher risk of invasion and metastasis. Therefore, the clinical significance of one factor (survivin or VEGF-C alone) may be affected by the expression of the other factor in the same patient. The combined analysis of associated factors may be of clinical significance for patients with gastric cancer.

To further investigate the biological co-effects of survivin and VEGF-C in gastric cancer, the mRNA and protein expression levels of survivin and VEGF-C were analyzed in the SGC7901 human gastric cancer cell line. Survivin and VEGF-C were highly expressed at the mRNA and protein levels. The proliferation and invasion of gastric cancer cells in vitro were significantly suppressed following silencing of survivin and VEGF-C by plasmids with survivin-shRNA and VEGF-C-shRNA. Moreover, the levels of VEGF-C expression were controlled by survivin, whereby the levels of survivin expression were not regulated by VEGF-C. Thus, there must be a hypothetical mechanism for how survivin controls the expression of VEGF-C.

Studies have demonstrated that phosphoinositide 3-kinase (PI3K)/Akt activation is involved in the upregulation of survivin $(25,26)$. The survivin mRNA expression levels are correlated with Akt activation, suggesting that Akt may be a downstream target (27). It has been demonstrated that the levels of survivin expression are regulated by the direct effect of PI3K alone via the PI3K/Akt signaling pathway in breast cancer cells (28). By contrast, VEGF-C expression is also correlated with the PI3K/Akt pathway in numerous types of cancer cells $(29,30)$. It is possible that survivin may regulate VEGF-C expression through the PI3K/Akt pathway.

COX-2 activation is highly correlated with survivin expression in certain types of tumor cells $(31,32)$ and, as an important regulator of apoptosis, COX-2 is able to upregulate the expression levels of survivin in cancer cells. VEGF-C expression is also regulated by COX-2. The COX-2 inhibitor mediates VEGF-C to block lymphangiogenesis and lymph node metastasis (33). It has also been proven that COX-2 is usually overexpressed with VEGF-C in breast, prostate and gastric cancer (34-36). Thus, COX-2 may be important in linking survivin and VEGF-C expression.

In the present study, following incubation in vitro with VEGF-C, SGC-7901 cells showed a significantly increased invasive ability. Moreover, the invasiveness of the gastric cancer cells significantly decreased when the survivin expression levels were downregulated. Thus, survivin may be important in enhancing the invasiveness of tumor cells initially caused by VEGF-C. The results demonstrate that VEGF-C protein is able to promote gastric cancer cell invasion and proliferation and suggest that the expression of VEGF-C in human gastric cancer cells may be regulated by survivin. They also indicate that VEGF-C-mediated tumor proliferation and metastases may be used in therapeutics by targeting survivin.

In conclusion, this study showed that survivin and VEGF-C were expressed in human gastric cancer cells, and that they were significantly associated with lymphatic metastasis. Patients with high expression levels of survivin and VEGF-C exhibited significantly less favorable survival rates compared with patients with low expression levels of those two genes. However, multivariate analysis demonstrated that neither of the two genes was an independent prognostic determinant. Survivin may promote VEGF-C expression through the PI3K/Akt pathway or COX-2 gene expression. Survivin may be a regulator of $\mathrm{VEGF}-\mathrm{C}$ expression in gastric cancer cells. Moreover, the study also provides a possible therapeutic method for the inhibition of VEGF-C-mediated tumor growth and lymphatic metastases by using a survivin-specific inhibitor.

\section{References}

1. Ferlay J, Shin HR, Bray F, Forman D, Mathers C and Parkin DM: Estimates of worldwide burden of cancer in 2008: GLOBOCAN 2008. Int J Cancer 127: 2893-2917, 2010.

2. Iida A, Hirose K, Arai M, Yamaguchi A and Nakagawara G: Relationships among the expression of epidermal growth factor receptor, proliferating cell nuclear antigen labeling index, and lymph node metastasis in gastric cancer. Oncology 52: 189-195, 1995.

3. Murata H, Kawano S, Tsuji S, et al: Cyclooxygenase-2 overexpression enhances lymphatic invasion and metastasis in human gastric carcinoma. Am J Gastroenterol 94: 451-455, 1999.

4. Yonemura Y, Endo Y, Fujita H, et al: Role of vascular endothelial growth factor $\mathrm{C}$ expression in the development of lymph node metastasis in gastric cancer. Clin Cancer Res 5: 1823-1829, 1999.

5. Miyachi K, Sasaki K, Onodera S, et al: Correlation between survivin mRNA expression and lymph node metastasis in gastric cancer. Gastric Cancer 6: 217-224, 2003.

6. Srinivasula SM and Ashwell JD: IAPs: what's in a name? Mol Cell 30: 123-135, 2008.

7. Altieri DC: The molecular basis and potential role of survivin in cancer diagnosis and therapy. Trends Mol Med 7: 542-547, 2001.

8. Wang TT, Qian XP and Liu BR: Survivin: potential role in diagnosis, prognosis and targeted therapy of gastric cancer. World J Gastroenterol 13: 2784-2790, 2007.

9. Chandele A, Prasad V, Jagtap JC, Shukla R and Shastry PR: Upregulation of survivin in $\mathrm{G} 2 / \mathrm{M}$ cells and inhibition of caspase 9 activity enhances resistance in staurosporine-induced apoptosis. Neoplasia 6: 29-40, 2004.

10. Da CL, Xin Y, Zhao J and Luo XD: Significance and relationship between Yes-associated protein and survivin expression in gastric carcinoma and precancerous lesions. World J Gastroenterol 15: 4055-4061, 2009.

11. Xiaoyuan C, Longbang C, Jinghua W, et al: Survivin: a potential prognostic marker and chemoradiotherapeutic target for colorectal cancer. Ir J Med Sci 179: 327-335, 2010.

12. Mandriota SJ, Jussila L, Jeltsch M, et al: Vascular endothelial growth factor-C-mediated lymphangiogenesis promotes tumour metastasis. EMBO J 20: 672-682, 2001. 
13. Kawakami M, Yanai Y, Hata F and Hirata K: Vascular endothelial growth factor $\mathrm{C}$ promotes lymph node metastasis in a rectal cancer orthotopic model. Surg Today 35: 131-138, 2005.

14. Stacker SA, Achen MG, Jussila L, Baldwin ME and Alitalo K Lymphangiogenesis and cancer metastasis. Nat Rev Cancer 2: 573-583, 2002.

15. Shida A, Fujioka S, Kobayashi K, et al: Expression of vascular endothelial growth factor (VEGF)-C and -D in gastric carcinoma. Int J Clin Oncol 11: 38-43, 2006.

16. Dikken JL, van de Velde CJ, Gönen M, et al: The New American Joint Committee on Cancer/International Union Against Cancer staging system for adenocarcinoma of the stomach: increased complexity without clear improvement in predictive accuracy. Ann Surg Oncol 19: 2443-2451, 2012.

17. Chomczynski P and Sacchi N: Single-step method of RNA isolation by acid guanidinium thiocyanate-phenol-chloroform extraction. Anal Biochem 162: 156-159, 1987.

18. Pennati M, Folini $\mathrm{M}$ and Zaffaroni N: Targeting survivin in cancer therapy. Expert Opin Ther Targets 12: 463-476, 2008.

19. Al-Joudi FS, Iskandar ZA, Hasnan J, et al: Expression of survivin and its clinicopathological correlations in invasive ductal carcinoma of the breast. Singapore Med J 48: 607-614, 2007.

20. Kim YH, Kim SM, Kim YK, Hong SP, Kim MJ and Myoung H: Evaluation of survivin as a prognostic marker in oral squamous cell carcinoma. J Oral Pathol Med 39: 368-375, 2010.

21. Su L, Wang Y, Xiao M, Lin Y and Yu L: Up-regulation of survivin in oral squamous cell carcinoma correlates with poor prognosis and chemoresistance. Oral Surg Oral Med Oral Pathol Oral Radiol Endod 110: 484-491, 2010.

22. Ryan BM, O'Donovan N and Duffy MJ: Survivin: a new target for anti-cancer therapy. Cancer Treat Rev 35: 553-562, 2009.

23. Makinen T, Jussila L, Veikkola T, et al: Inhibition of lymphangiogenesis with resulting lymphedema in transgenic mice expressing soluble VEGF receptor-3. Nat Med 7: 199-205, 2001.

24. Wirzenius M, Tammela T, Uutela M, et al: Distinct vascular endothelial growth factor signals for lymphatic vessel enlargement and sprouting. J Exp Med 204: 1431-1440, 2007.

25. Ding S, Li C, Lin S, et al: Distinct roles of VEGF-A and VEGF-C in tumour metastasis of gastric carcinoma. Oncol Rep 17: 369-375, 2007

26. Papapetropoulos A, Fulton D, Mahboubi K, et al: Angiopoietin-1 inhibits endothelial cell apoptosis via the Akt/survivin pathway. J Biol Chem 275: 9102-9105, 2000.
27. Mesri M, Morales-Ruiz M, Ackermann EJ, et al: Suppression of vascular endothelial growth factor-mediated endothelial cell protection by survivin targeting. Am J Pathol 158: 1757-1765, 2001.

28. Zhao P, Meng Q, Liu LZ, You YP, Liu N and Jiang BH: Regulation of survivin by PI3K/Akt/p70S6K1 pathway. Biochem Biophys Res Commun 395: 219-224, 2010.

29. Asanuma H, Torigoe T, Kamiguchi K, et al: Survivin expression is regulated by coexpression of human epidermal growth factor receptor 2 and epidermal growth factor receptor via phosphatidylinositol 3-kinase/AKT signaling pathway in breast cancer cells. Cancer Res 65: 11018-11025, 2005.

30. Tsutsui S, Matsuyama A, Yamamoto M, et al: The Akt expression correlates with the VEGF-A and -C expression as well as the microvessel and lymphatic vessel density in breast cancer. Oncol Rep 23: 621-630, 2010.

31. Xiang L, Xie G, Ou J, Wei X, Pan F and Liang H: The extra domain A of fibronectin increases VEGF-C expression in colorectal carcinoma involving the PI3K/AKT signaling pathway. PLoS One 7: e35378, 2012.

32. Song IH, Kim DW, Shin KC, et al: Down-regulation of survivin in growth inhibition of hepatoma cells induced by a selective cyclooxygenase-2 inhibitor. Korean J Hepatol 14: 351-359, 2008 (In Korean).

33. Jang JW: Anti-tumor mechanisms and regulation of survivin by selective cyclooxygenase-2 inhibitor. Korean J Hepatol 14: 305-308, 2008 (In Korean).

34. Iwata C, Kano MR, Komuro A, et al: Inhibition of cyclooxygenase-2 suppresses lymph node metastasis via reduction of lymphangiogenesis. Cancer Res 67: 10181-10189, 2007.

35. Bhattacharjee RN, Timoshenko AV, Cai J and Lala PK Relationship between cyclooxygenase- 2 and human epidermal growth factor receptor 2 in vascular endothelial growth factor $\mathrm{C}$ up-regulation and lymphangiogenesis in human breast cancer. Cancer Sci 101: 2026-2032, 2010.

36. Di JM, Zhou J, Zhou XL, et al: Cyclooxygenase-2 expression is associated with vascular endothelial growth factor-C and lymph node metastases in human prostate cancer. Arch Med Res 40: 268-275, 2009.

37. Zhang J, Ji J, Yuan F, et al: Cyclooxygenase-2 expression is associated with VEGF-C and lymph node metastases in gastric cancer patients. Biomed Pharmacother 59 (Suppl 2): S285-S288, 2005. 